Савцова Анна Валерьевна, Гринь Анна Александровна, Гринь Александр Иванович, Скороходова Ирина Георгиевна, Волков Сергей Михайлович

\title{
РАЗРАБОТКА АЛГОРИТМА ДЛЯ ИССЛЕДОВАНИЯ ВЛИЯНИЯ ПОГРЕШНОСТЕЙ ЗАДАНИЯ СХЕМНОЙ И РЕЖИМНОЙ ИНФОРМАЦИИ НА ВЕЛИЧИНУ ПОТЕРЬ МОЩНОСТИ
}

Статья посвящена актуальной на сегодняшний день проблеме исследования влияния погрешности задания схемной и режимной информации на величину потерь моциости в питаюцих и распределительных сетях высокого напряжсени сетях сетевых компаний. Авторами предложен способ оченки влияния неточности задания режимных и схемных параметров на величину потерь моцности и на эффективность выбора мероприятий по снижению потерь электроэнергии (ЭЭ), основанный на использовании имитационного моделирования. Выполнена оченка степени влияния погрешностей задания схемной и режсимной информации на результаты расчетов потерь моцности в сетях 110 кВ для двух филиалов ПАО «МРСК Северного Кавказа»-Россети.

Ключевые слова: погречности, методика исследования погрешностей, потери моцности, питающая сеть.

Anna Savtsova, Anna Grin, Alexander Grin, Irina Skorohodova, Sergey Volkov

DEVELOPMENT OF THE ALGORITHM FOR INVESTIGATION

OF THE INFLUENCE OF THE ERRORS OF THE REQUIREMENT

OF THE DIAGNOSTIC AND REGIME INFORMATION

ON THE VALUE OF POWER LOSS

The article is devoted to the actual problem of studying the influence of the error in setting schematic and regime information on the amount of power losses in high voltage feed and distribution networks of grid companies. The authors proposed a method for estimating the influence of inaccuracy in setting the mode and circuit parameters on the amount of power losses and on the efficiency of choosing measures to reduce energy losses, based on the use of simulation modeling. The estimation of the degree of influence of errors in the specification of the circuit and mode information on the results of power loss calculations in $110 \mathrm{kV}$ networks for two branches of IDGC of the North Caucasus, JSC - Rosseti, was made.

Key words: of error, error investigation technique, power loss, power supply network.

Bведение / Introduction. Несмотря на усилия сетевых компаний по снижению потерь ЭЭ и принятие ряда нормативных документов, за последние годы не произошло существенного снижения потерь ЭЭ в сетях сетевых компаний. В первую очередь это связано с трудностью расчета достоверной величины потерь ЭЭ из-за информационной недостаточности и существующих погрешностей задания схемных и режимных параметров, а также отсутствия заинтересованности сетевых компаний в реальном существенном снижении потерь ЭЭ ввиду несовершенства методик нормирования технологических потерь.

Основными причинами, влияющими на эффективность выбора мероприятий по снижению потерь (МСП) ЭЭ, являются:

- сложность учета температурного влияния на сопротивления элементов схемы;

- пренебрежение распределенностью и волновым характером параметров линии;

- принятие неизменности схемы сети на расчетном периоде времени;

- наличие погрешности замеров режимных параметров и определения схемных параметров сети;

- отсутствие необходимой информации для выбора мероприятий на расчетных интервалах времени и т. д. 
Количественная оценка погрешностей методов расчета потерь сопряжена с весьма серьезными трудностями, так как натурный эксперимент в реальной сети значительного объема и на большом интервале времени выполнить практически невозможно из-за необходимых для этой цели трудозатрат по проведению измерений и обработки результатов.

Выбор МСП связан с анализом больших объемов режимной информации, получаемой при расчетах потокораспределения в сети по данным сезонных контрольных замеров. В этом случае методические погрешности расчета менее важны, чем инструментальные, так как эффект от снижения потерь ЭЭ рассчитывается, как разность потерь до и после реализации мероприятия.

Эффективность разрабатываемых мероприятий в значительной степени зависит от учета изменения схемы сети и параметров режима в течение расчетного периода. Кроме того, на выбор режимных мероприятий существенное влияние оказывают погрешности определения как параметров режима, так и параметров схемы.

Наличие погрешности расчета потерь ЭЭ обусловлено приближенной математической моделью, погрешностями измерения или расчета параметров режима и погрешностями применяемых методов расчета. Составляющие результирующей погрешности обусловлены случайным характером нагрузок потребителей, внешним воздействием на параметры сети и параметры режима.

На практике активные и реактивные сопротивления ветвей, активные и реактивные проводимости элементов сети на землю, коэффициенты трансформации измеряются со значительными погрешностями [1-6].

Наибольшее влияние на погрешности определения параметров схемы замещения оказывают погодные условия, ошибки в определении длин линий, изменения номинальных параметров трансформаторов после капремонтов и др.

Основной гипотезой данного исследования является предположение о том, что выявление степени влияния погрешности задания различных параметров на величину суммарных потерь мощности будет способствовать наиболее достоверному выбору МСП ЭЭ.

В силу указанных обстоятельств было принято решение о разработке алгоритма и программного обеспечения для количественной оценки влияния погрешности задания схемных и режимных параметров на погрешность расчета потерь мощности.

Maтериалы и методы /Materials and methods. В качестве объекта исследования выбрана питающая сеть напряжением 110 кВ двух сетевых компаний ПАО МРСК Северного Кавказа и фидера 6-10 кВ АО Горэлектросеть г. Ставрополя. Характеристика расчетных схем представлена в таблице 1 .

Таблица 1

Характеристика исследуемых схем

\begin{tabular}{|c|l|l|}
\hline $\begin{array}{c}\text { № } \\
\text { схемы }\end{array}$ & \multicolumn{1}{|c|}{ Исследуемая сеть } & \multicolumn{1}{|c|}{ Примечание } \\
\hline 1 & $\begin{array}{l}\text { Кабардино-Балкарский филиал МРСК Северного } \\
\text { Кавказ (КБФ) }\end{array}$ & $\begin{array}{l}\text { Средняя РСК с эталонными потерями } \\
\text { в 33 Мвт }\end{array}$ \\
\hline 2 & $\begin{array}{l}\text { Карачаево-Черкесский филиал МРСК Северного } \\
\text { Кавказ (КЧФ) }\end{array}$ & $\begin{array}{l}\text { Небольшая РСК с эталонными потерями } \\
\text { в 9 Мвт }\end{array}$ \\
\hline 3 & $\begin{array}{l}\text { АО «Ставропольские городские электрические } \\
\text { сети» }\end{array}$ & Территориальная сетевая компания \\
\hline
\end{tabular}

Расчеты потерь в сети 110 кВ выполнялись по полным схемам замещения, используемым в РДУ Северо-Кавказского филиала СО «ЦДУ ЕЭС» для обработки контрольных замеров в режимные дни. Расчетные схемы сети 110 кВ включают все замкнутые контуры.

Для исследования влияния составляющих результирующей погрешности предлагается алгоритм, блок-схема которого представлена на рис. 1. 


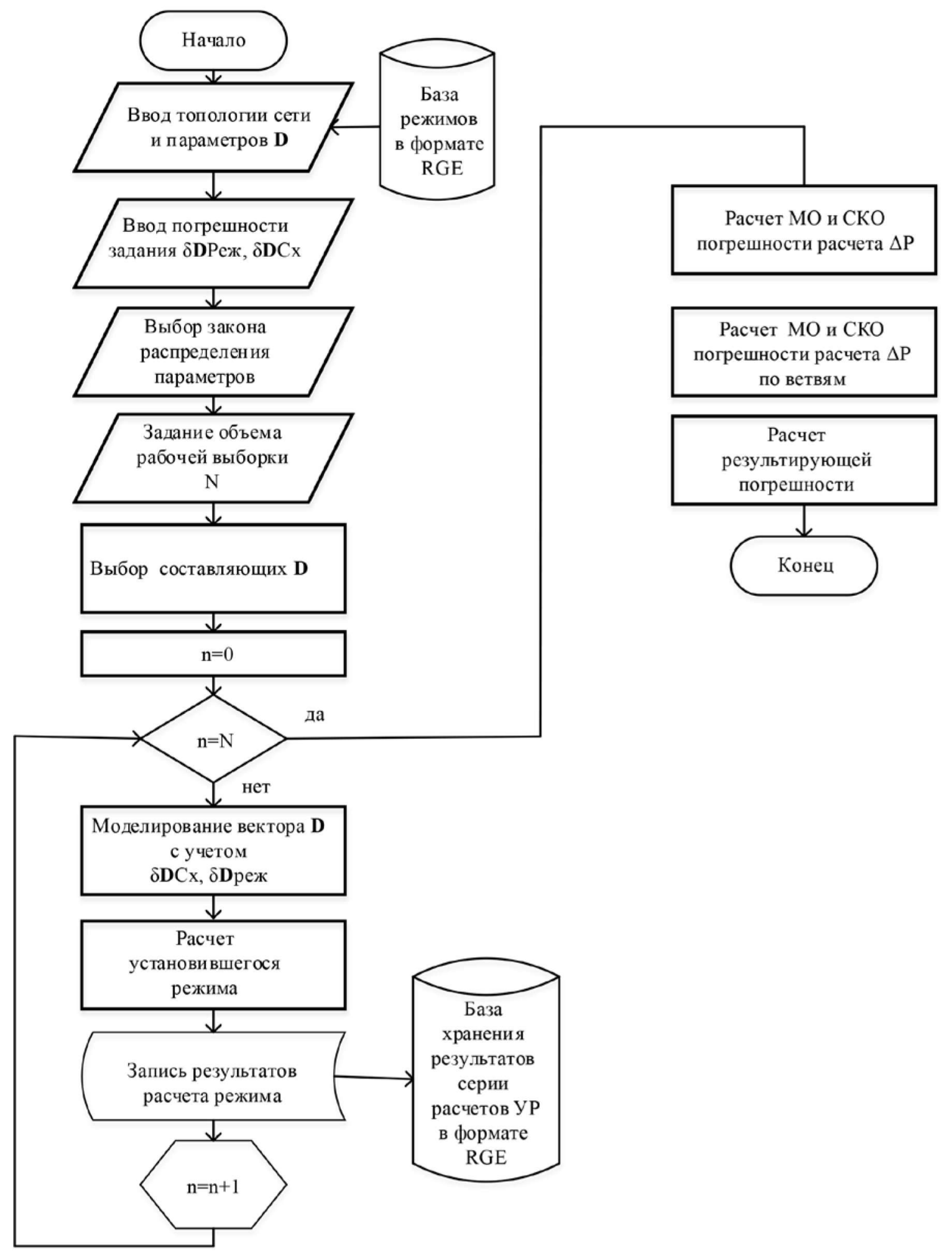

Рис. 1. Блок-схема алгоритма исследования составляющих результирующей погрешности 
Предлагаемый алгоритм позволяет с качественной и количественной стороны оценить степень влияния различных параметров или их сочетаний на величину потерь мощности.

Для количественного изучения влияния каждой составляющей на результирующую погрешность расчета потерь мощности предлагается осуществить действия в следующей последовательности:

- выявить диапазоны возможных значений погрешностей определения режимных и схемных параметров;

- в качестве условно-эталонной модели принять результаты расчета потерь мощности по промышленной программе расчета установившегося режима при задании в узлах математических ожиданий нагрузок за исследуемый период времени;

- задаться доверительной вероятностью;

- рассчитать серию установившихся режимов для моделирования отклонений схемных и режимных параметров по нормальному или равномерному законам;

- рассчитать погрешность определения потерь мощности обусловленную составляющими результирующей погрешности;

- определить отдельно степень влияния каждого из режимных и схемных параметров на результаты расчета потерь мощности.

В качестве условно-эталонной математической модели будем понимать такую математическую модель, свойства которой максимально совпадают со свойствами реальной сети. В данном случае предлагается использовать решение векторного уравнения:

$$
\underline{\mathrm{W}}\left(Z, \underline{\mathrm{S}}_{\mathrm{H}}(Z), \underline{\mathrm{Y}}_{\mathrm{y}}\right)=0,
$$

где $\underline{\mathrm{W}}$ - комплексный вектор небалансов мощностей в узлах электрической сети; $\underline{\mathrm{S}}_{\mathrm{H}}(\mathrm{Z})$ - комплексный вектор мощностей нагрузок в узлах; $\underline{Y}_{y}$ - матрица узловых проводимостей.

Потери мощности при использовании выражения в виде квадратичной формы от узловых напряжений, рассчитываются в виде разности между мощностями генераторов и нагрузок в узлах:

$$
\Delta \underline{S}_{\Sigma}=\sum_{\mathrm{k}=1}^{\mathrm{n}+1} \mathrm{~S}_{\mathrm{k}}=\sum_{\mathrm{k}=1}^{\mathrm{n}+1} \sqrt{3} \underline{\mathbf{U}}_{\mathrm{k}} \underline{\mathbf{I}}_{\mathrm{k}}^{*}
$$

где $\Delta \underline{S}_{\Sigma}-$ суммарные потери в продольной и поперечной частях схемы сети.

Обозначим вектор точных исходных данных символом $\bar{D}$. Соответствующая этому вектору неявная вектор-функция $F(\bar{D})$ определяет величину потерь мощности.

Погрешность результата, внесенную прерыванием итерационного процесса при расчете установившегося режима, определим по выражению

$$
\delta_{\mu}=F(\bar{D})-F(\bar{D}, \alpha),
$$

где $F(\bar{D}, \alpha)$ - вектор-функция потерь мощности, полученная с учетом погрешности прерывания итерационного процесса при расчете установившегося режима.

Погрешность использования приближенной модели определим по выражению

$$
\delta_{\mu}=F(\bar{D})-F(\bar{D}, \alpha, \mu),
$$

где $F(\bar{D}, \alpha, \mu)$ - вектор-функция потерь мощности, полученная с учетом погрешности прерывания итерационного процесса и модели.

Погрешность модели обусловлена изменением схемы сети в условиях эксплуатации в течение расчетного периода.

Погрешность определения вектор-функции потерь мощности из-за погрешностей исходных данных предлагается определять по выражению

$$
\delta_{D}=F(\bar{D})-F(\bar{D}, \alpha, \mu),
$$

где $F(\bar{D})$ - вектор-функция потерь мощности, полученная с учетом погрешности исходных данных. 
Разработанный алгоритм позволяет исследовать различные погрешности в условиях, когда имеет место только данная погрешность, а все другие погрешности отсутствуют, либо оценить влияние нескольких составляющих результирующей погрешности. С его помощью можно перейти к нормам погрешностей вектора по группам компонент вида:

$$
\|\delta\| \mathrm{i}=\frac{1}{\mathrm{~N}} \sum_{\mathrm{i}=1}^{\mathrm{N}}\left|\delta_{i}\right| .
$$

Используя предлагаемый алгоритм, можно рассчитать доверительный интервал как интервал, окружающий оценку, таким образом, чтобы с определенной вероятностью истинное значение находилось внутри этого интервала.

Оценка погрешностей производилась по формуле

$$
\delta_{i}=\frac{t \cdot \mu}{M O} \cdot 100 \%,
$$

где $t$ - коэффициент Стьюдента; $\mu$ - ошиб́ка выборки, определяемая по формуле:

$$
\mu=\frac{\sigma_{t}}{\sqrt{n}} \text {. }
$$

где $\sigma_{t}-$ величина среднеквадратического отклонения, рассчитанная по выборке.

Объем выборки при проведении исследований принят равным 100 расчетам установившихся режимов при различных погрешностях исходных данных.

Результаты и обсуждение/Results and discussion. В результате поискового эксперимента было установлено, что точность расчета режима по мощности для прекращения итерационного процесса должна быть не больше $0,001 \mathrm{MBT}$, при этом погрешность от прерывания итерационного процесса при расчете установившегося режима не превышает $0,00007 \%$.

При исследовании питающих сетей рассматривались диапазоны относительных погрешностей представленных в таблице 2 [1-6].

Диапазоны изменения схемных и режимных параметров питающей сети

\begin{tabular}{|l|c|c|c|c|c|c|c|c|c|c|}
\hline \multicolumn{1}{|c|}{ Параметр } & $\boldsymbol{R}_{\Pi}$ & $\boldsymbol{X}_{J}$ & $\boldsymbol{B}_{J}$ & $\boldsymbol{G}_{J}$ & $\boldsymbol{R}_{T}$ & $\boldsymbol{X}_{T}$ & $\boldsymbol{B}_{T}$ & $\boldsymbol{G}_{T}$ & $\boldsymbol{P}_{n}$ & $\boldsymbol{Q}_{u}$ \\
\hline $\begin{array}{l}\text { Диапазоны исследуемых } \\
\text { погрешностей }\end{array}$ & \pm 12 & \pm 8 & \pm 20 & \pm 200 & \pm 16 & \pm 16 & \pm 25 & \pm 40 & \pm 10 & \pm 15 \\
\hline
\end{tabular}

На рисунках 2-5 представлены полученные зависимости влияния погрешностей задания схемной и режимной информации на величину потерь мощности для двух сетевых компаний.

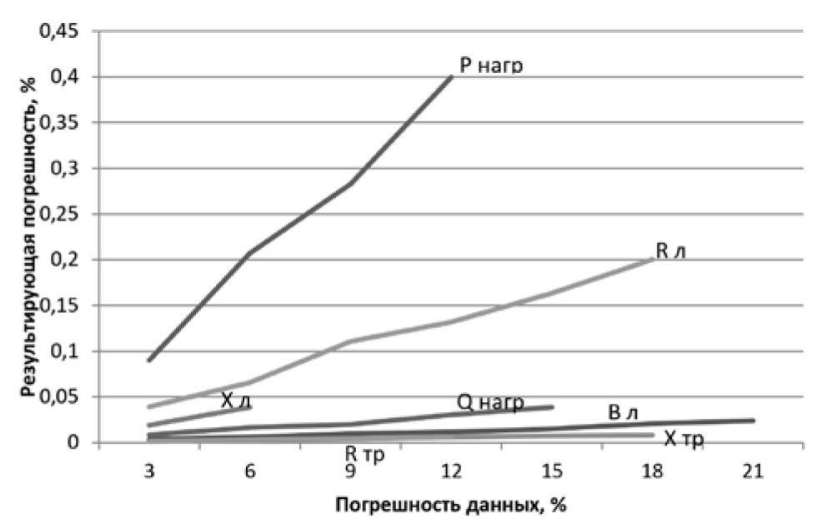

Рис. 2. Зависимости погрешности расчета потерь мощности от погрешностей задания вектора D для КЧФ МРСК Северного Кавказ при моделировании по нормальному закону распределения 


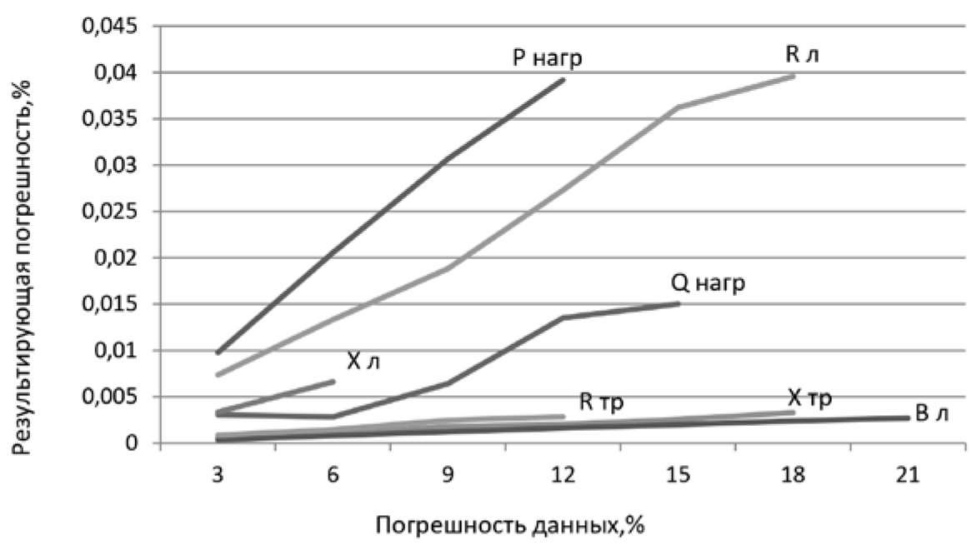

Рис. 3. Зависимости погрешности расчета потерь мощности от погрешностей задания вектора D для КЧФ МРСК Северного Кавказа при моделировании по равномерному закону распределения

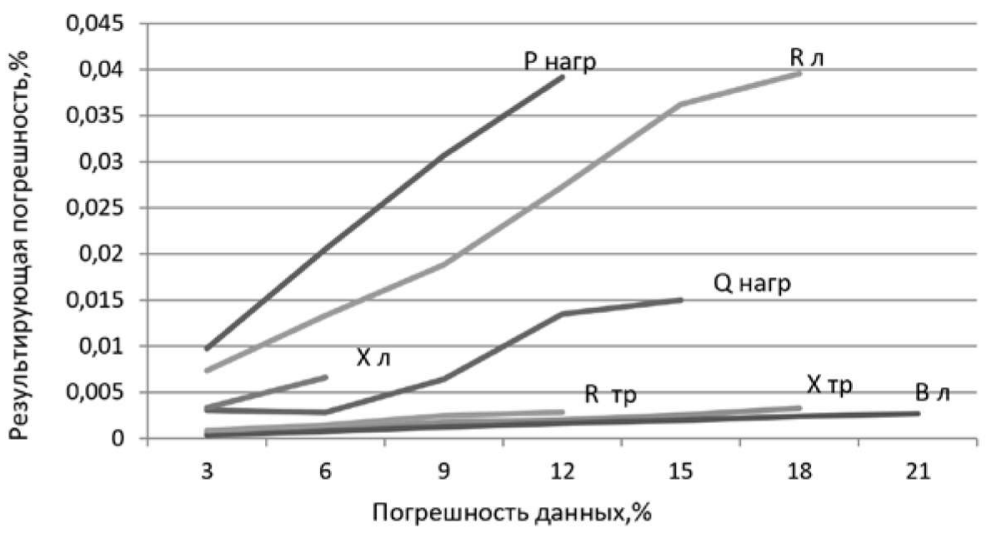

Рис. 4. Зависимости погрешности расчета потерь мощности от погрешностей задания вектора D для КБФ МРСК Северного Кавказа при моделировании по нормальному закону распределения

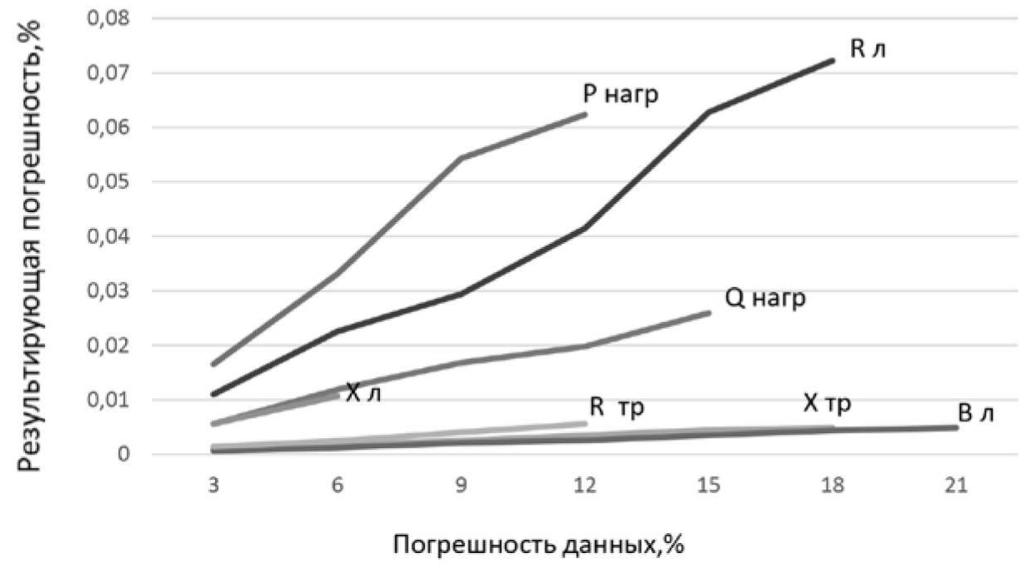

Рис. 5. Зависимости погрешности расчета потерь мощности от погрешностей задания вектора D для КБФ МРСК Северного Кавказа при моделировании по равномерному закону распределения 
Анализ рисунков 2-5 позволяет сделать вывод, что на результирующую погрешность наибольшее влияние оказывает погрешность задания активной нагрузки и активных сопротивления линий.

Исследование совместного взаимодействия всех рассматриваемых погрешностей показало, что погрешность расчета суммарных потерь мощности не превышает $1,2 \%$, а погрешность расчета потерь мощности по ветвям схемы может достигать $200 \%$.

При использовании данного алгоритма объектом исследования распределительной сети являлись схемные и режимные параметры, представленные в таблице 3.

Таблица 3

\section{Диапазоны изменения схемных и режимных параметров распределительной сети 6-10 кВ}

\begin{tabular}{|l|c|c|c|c|c|c|c|c|c|c|}
\hline \multicolumn{1}{|c|}{ Параметр } & $\boldsymbol{R}_{I}$ & $\boldsymbol{X}_{J}$ & $\boldsymbol{B}_{J}$ & $\boldsymbol{G}_{J}$ & $\boldsymbol{R}_{T}$ & $\boldsymbol{X}_{T}$ & $\boldsymbol{B}_{T}$ & $\boldsymbol{G}_{T}$ & $\boldsymbol{P}_{\boldsymbol{r}}$ & $\boldsymbol{Q}_{\mu}$ \\
\hline $\begin{array}{l}\text { Диапазоны исследуемых } \\
\text { погрешностей }\end{array}$ & \pm 16 & \pm 4 & \pm 20 & \pm 25 & \pm 12 & \pm 17 & \pm 10 & \pm 10 & \pm 10 & \pm 15 \\
\hline
\end{tabular}

В качестве условно-эталонной модели принят расчет установившегося режима по программе RERS PC [7] при задании в узлах средних нагрузок. В качестве примера рассмотрено несколько фидеров, данные о которых представлены в таблице 4.

Исследуемые фидера сети 6-10 кВ

Таблица 4

\begin{tabular}{|c|l|c|c|}
\hline Номер фидера & \multicolumn{1}{|c|}{ Название фидера } & Средний ток, А & $\begin{array}{c}\text { Эталонное значение } \\
\text { потерь, кBт }\end{array}$ \\
\hline 1 & Восточная $\Phi-689$ & 84 & 20,3 \\
\hline 2 & Западная $\Phi-669$ & 597 & 195,0 \\
\hline 3 & Промышленная $\Phi-646$ & 15 & 11,2 \\
\hline 4 & Северная $\Phi-619$ & 687 & 451,5 \\
\hline 5 & Южная $\Phi 187$ & 95 & 96,83 \\
\hline
\end{tabular}

Погрешности определения потерь мощности при учете совокупности погрешностей параметров представлены в таблице 5 .

Результаты исследования погрешностей задания D в РС ВН

Таблица 5

\begin{tabular}{|c|c|c|c|c|c|c|c|}
\hline \multirow{2}{*}{$\begin{array}{c}\text { № } \\
\text { фидера }\end{array}$} & \multirow{2}{*}{ Наименование фидера } & \multicolumn{2}{|c|}{$\begin{array}{c}\text { Нормальное } \\
\text { распределение }\end{array}$} & \multicolumn{2}{|c|}{$\begin{array}{c}\text { Равномерное } \\
\text { распределение }\end{array}$} & \multirow{2}{*}{$\begin{array}{l}\text { Среднеквадратичная } \\
\text { погрешность, кBт }\end{array}$} & \multirow{2}{*}{$\begin{array}{l}\text { Результируюшая } \\
\text { погрешность, \% }\end{array}$} \\
\hline & & МО, кВт & СКО, кВт & МO, кBт & СКО, кВТ & & \\
\hline 1 & Восточная Ф-689 & 0,0195 & 1,51 & 0,05 & 1,14 & 0,107 & 1,58 \\
\hline 2 & Западная Ф-669 & 4,00 & 33,7 & 1,8 & 20,98 & 2,383 & 3,67 \\
\hline 3 & Промышленная Ф-646 & 0,035 & 1,95 & 0,089 & 1,29 & 0,138 & 3,69 \\
\hline 4 & Северная Ф-619 & 16,35 & 106,58 & 3,4 & 57,58 & 7,536 & 5,01 \\
\hline 5 & Южная Ф 187 & 0,18 & 2,73 & 0,164 & 1,84 & 0,193 & 0,60 \\
\hline
\end{tabular}

Заключение / Conclusion. Разработан алгоритм и программное обеспечение, необходимые для исследования влияния погрешностей задания схемных и режимных параметров при расчетах потерь мощности и разработке мероприятий по снижению потерь ЭЭ.

1. Установлено, что точность расчета установившегося режима по мощности должна быть не больше 0,001 МВт. 
2. Разработанный алгоритм и программное обеспечение позволят при проведении сравнительного анализа различных мероприятий по снижению потерь ЭЭ выявить влияния различных погрешностей на величину потерь мощности.

3. Показано, что погрешность расчета суммарных потерь мощности не превышает $1,2 \%$, а погрешность расчета потерь мощности по ветвям схемы может достигать $200 \%$.

4. Необходимы дальнейшие исследования, направленные на установление диапазонов отдельных составляющих и выявление степени изменения величины потерь ЭЭ из-за изменения топологии схемы сети.

\section{ЛИТЕРАТУРА И ИНТЕРНЕТ-РЕСУРСЫ}

1. Идельчик В. И. Расчеты и оптимизация режимов электрических сетей и систем. М.: Энергоатомиздат, 1988. $288 \mathrm{c}$.

2. Методы оперативной идентификации параметров схем замещения элементов электроэнергетических систем / А. С. Бердин, П. А. Крючков, А. А. Суворов, С. Н. Шелюг // Конференция молодых специалистов электроэнергетики -2000: сб. докл. М.: НЦЭНАС, 2000. С. 153-155.

3. Глущик А. Е. Анализ режимных параметров и потерь электроэнергии в сети 6-10 кВ г. Ставрополя (научная статья)/ А. Е. Глущик, А. И. Гринь, А. А. Гринь, И. Г. Скороходова, А. А. Зубков // Сборник научных трудов по материалам $V$ ежегодной научно-практической конференции Северо-Кавказского федерального университета «Университетская наука - региону» (Ставрополь, 2017). Ставрополь: ООО ИД ТЭСЭРА, 2017. $345 \mathrm{c}$.

4. Железко Ю. С., Артемьев А. В., Савченко О. В. Расчет, анализ и нормирование потерь электроэнергии в электрических сетях: практическое пособие. М.: НЦ ЭНАС, 2006. 280 с.

5. Заславская Т. Б., Ирлахман М. Я. Пределы вариации электрических параметров силовых трансформаторов // Работы в области электроэнергетических систем: Труды СибНИИЭ. Вып. 20. М.: Энергия, 1971. С. 114-117.

6. Идельчик В. И., Новиков А. С., Паламарчук С. И. Ошибки задания параметров схем замещения при расчетах режимов электрических систем // Статистическая обработка оперативной информации в электроэнергетических системах. Иркутск, 1979. С. 145-152.

7. Кононов Ю. Г., Кононов А. Ю., Кононов А. Ю. Комплекс программ для решения режимно-технологических задач в электрических сетях «RersPC» (комплекс «RersPC»): Свидетельство об официальной регистрации программы для ЭВМ № 2005610771 (31.03.2005) // Программы для ЭВМ, базы данных, топологии интегральных микросхем. М: ФИПС. 2005. № 2. С. 179.

\section{REFERENCES AND INTERNET RESOURCES}

1. Idelchik V. I. Calculations and optimization of modes of electrical networks and systems. - Moscow: Energoatomizdat, 1988. $-288 \mathrm{p}$.

2. Methods of operative identification of the parameters of substitution schemes for electric power system elements / A. S. Berdin, P. A. Kryuchkov, A. A. Suvorov, S. N. Shelyug // Conference of young specialists in electric power industry - 2000: Sat. doc. M.: NCEENAS, 2000. Pp. 153-155.

3. Glushik A. E. Analysis of regime parameters and power losses in a 6-10 kV network in the city of Stavropol (scientific article)/ A. E. Glushik, A. I. Grin, A. A. Grin, I. G. Skorokhodova, A. A. Zubkov // Collection of proceedings on the materials of $V$ annual scientific and practical conference of the North-Caucasian Federal University «University Science - Region» (Stavropol, 2017). Stavropol: Open Company ID TESERA, 2017. 345 p.

4. Zhelezko Yu. S., Artemiev A. V., Savchenko O. V. Calculation, analysis and normalization of electric power losses in electric networks: pract. allowance. М.: НЦ ЭНАC, 2006. $280 \mathrm{c}$.

5. Zaslavskaya T. B., Irlakhman M. Ya. Limits of variation of electrical parameters of power transformers // Works in the field of electric power systems: Proceedings of SibNIIE. Issue. 20. M.: Energia, 1971. Pp. 114-117. 
6. Idelchik V. I., Novikov A. S., Palamarchuk S. I. Errors in specifying the parameters of substitution circuits for calculating the modes of electrical systems // Statistical processing of operational information in electric power systems. Irkutsk, 1979. Pp. 145-152.

7. Kononov Yu. G., Kononov A. Yu., Kononov A. Yu. A set of programs for solving the regime-technological problems in the electrical networks «RersPC» (complex «RersPC»): Certificate of the official registration of the computer program № 2005610771 (31.03.2005) // Computer programs, databases, topographies of integrated microcircuits. M: FIPS. 2005. No. 2. P. 179.

\section{СВЕДЕНИЯ ОБ АВТОРАХ}

Савцова Анна Валерьевна, доктор экономических наук, доцент, профессор кафедры финансов и кредита Северо-Кавказского федерального университета. E-mail: levandanna@yandex.ru

Гринь Анна Александровна, кандидат экономических наук, доцент кафедры автоматизированных электроэнергетических систем и электроснабжения Северо-Кавказского федерального университета. E-mail: agrin22@mail.ru

Гринь Александр Иванович, кандидат технических наук, доцент кафедры автоматизированных электроэнергетических систем и электроснабжения Северо-Кавказского федерального университета. E-mail: aigrin@ ncfu.ru

Скороходова Ирина Георгиевна, кандидат экономических наук, доцент кафедры экономики и внешнеэкономической деятельности Северо-Кавказского федерального университета. E-mail: iskorokhodova9@ gmail.com.

Волков Сергей Михайлович, аспирант кафедры АЭСиЭ Северо-Кавказского федерального университета. E-mail: sergey_volkov_92@list.ru

\section{INFORMATION ABOUT AUTHORS}

Savtzova Anna, Doctor of Economics, Associate Professor, Professor of the Department of Finance and Credit, North Caucasus Federal University. E-mail: levandanna $(a y a n d e x . r u$

Grin Anna, Ph.D., Associate Professor, Department of automated electric power systems and power the North Caucasus Federal University. E-mail: agrin22@mail.ru

Grin Alexander, Ph.D., Associate Professor, Department of automated electric power systems and power the North Caucasus Federal University. E-mail: aigrin@ncfu.ru

Skorokhodova Irina, Ph.D., assistant professor of economics and foreign trade activities of the North Caucasus Federal University. E-mail: iskorokhodova9@gmail.com

Volkov Sergey, graduate student, Department of automated electric power systems and power the North Caucasus Federal University. E-mail: sergey_volkov_92@list.ru. 